\title{
Lecturatón en la IByCENECH
}

\author{
Readathon at IByCENECH
}

\author{
Vianey Salinas Porras - Ruth Oros Macías - Coynta Becerra Aragonés
}

Vianey Salinas Porras. Institución Benemérita y Centenaria Escuela Normal del Estado "Profr. Luis Urías Belderráin", Chihuahua, México. Es licenciada en Educación Primaria, licenciada en Educación Secundaria con especialidad en Español, maestra en Pedagogía Terapéutica, maestra en Terapia Familiar Sistémica y doctorante en Psicoterapia Familiar y de Pareja. Labora como docente en la Institución Benemérita y Centenaria Escuela Normal del Estado de Chihuahua "Profr. Luis Urías Belderráin" y está a cargo del Departamento de Atención a Estudiantes. Es miembro del cuerpo académico BCENELUB-CA 6. Correo electrónico: v.salinas@ibycenech.edu.mx. ORCID: https://orcid.org/0000-0002-0048-8160.

Ruth Oros Macías. Institución Benemérita y Centenaria Escuela Normal del Estado "Profr. Luis Urías Belderráin", Chihuahua, México. Es licenciada en Educación Preescolar, licenciada en Historia, maestra en Educación y doctora en Educación. Ha cursado diplomados y talleres en diferentes áreas de atención a la educación especial. Desde el año 2013 labora como docente en la Institución Benemérita y Centenaria Escuela Normal del Estado "Profr. Luis Urías Belderráin". Forma parte del Cuerpo Académico BCENELUB-CA 6, desde donde realiza actividades de investigación. Correo electrónico: rn.oros@ ibycenech.edu.mx. ORCID: https://orcid.org/00000002-8853-0318.

Coynta Becerra Aragonés. Institución Benemérita y Centenaria Escuela Normal del Estado "Profr. Luis Urías Belderráin", Chihuahua, México. Es licenciada en Educación Primaria por la Normal del Estado de Chihuahua, licenciada en Educación por la Universidad Pedagógica Nacional y maestrante en Educación para el Desarrollo Profesional Docente en la Normal

\section{Resumen}

México, como muchos países, se vio envuelto en la vorágine de la experiencia que nos ofreció la COVID-19, sin embargo, como siempre que ha habido desastres, salimos adelante y esta ocasión no fue la excepción, así mismo la Institución Benemérita y Centenaria Escuela Normal del Estado de Chihuahua -IByCENECH- en estos momentos de crisis buscó cómo resolver y acrecentar las experiencias y aprendizajes de los alumnos, encontrando alternativas nuevas y diferentes a las que venía ofreciendo, por ello se da la implementación del proyecto "Lecturatón IByCENECH”, que da cabida a docentes, administrativos y alumnos de todas las licenciaturas, encontrando una respuesta favorable ante la convocatoria. La mecánica de participación consistió en hacer la lectura de libros y sus respectivas reseñas, en este proceso se vio favorecido el acercamiento a la lectura, la escritura y compartir con otros nuestra experiencia lectora, ese mundo de letras convertidas en historias con grandes mensajes. Este proyecto surge con un propósito explícito de fomentar el gusto por la lectura, además de ser una estrategia de autoayuda en tiempos de contingencia y otros implícitos para atender la parte en que la narrativa te envuelve, haciendo tu tiempo y espacio más placenteros.

Palabras clave: Lectura, comprensión lectora, autoayuda, narrativa.

Abstract
Mexico, like many countries, was involved in the whirlwind that the
COVID-19 experience gave us, however, as usual with disasters, we
got through and this occasion was not the exception. In the same way,
the Institución Benemérita y Centenaria Escuela Normal del Estado
-IByCENECH-, in these times of crisis, sought how to solve and
enhance the experiences and learning of the students, finding new and
different alternatives to those that had been offered. For this reason,
the implementation of the "IByCENECH Readathon" project-where
there is room for teachers, administrators, and students of all levels-
had a favorable response to the call. The participation rules consisted


Superior del Estado de Chihuahua. Cursó los diplomados en Educación Especial, Literatura Infantil Alemana, Lectura Creativa y Creación Literaria, y Escritura Autobiográfica. Es responsable de la biblioteca institucional de la IByCENECH y miembro activo del Club de Cuervos para lectores y escritores. Correo electrónico: c.becerra@ibycenech.edu.mx. https:// orcid.org/0000-0001-5839-2746. of reading books and writing their respective reviews. This process favored an approach to reading, writing, and sharing our reading experience to others, that world of letters turned into stories with great messages. This project arises with an explicit purpose of promoting the taste for reading, as well as being a self-help strategy in times of contingency and other implicit ones to attend the areas where narrative gets you involved, making time and space more pleasant.

Keywords: Reading, reading comprehension, self-help, narrative.

\section{INTRODUCCIÓN}

En la Institución Benemérita y Centenaria Escuela Normal del Estado de Chihuahua IByCENECH se implementó el proyecto “Lecturatón”, para sobrellevar de una manera óptima, funcional y enriquecedora el aislamiento derivado por la pandemia de la COVID-19 de marzo a junio del año 2020, con una competencia lectora, donde tuvo la oportunidad de participar la comunidad normalista de la institución, para acrecentar su acervo cultural y fomentar el gusto por la lectura; se motivó por medio de incentivos a los tres primeros lugares por las reseñas presentadas al mayor número de libros leídos.

Se aprovechó el uso de las redes sociales, creando un grupo cerrado en Facebook para dar seguimiento al reto y llevar un registro de los avances por medio de reseñas de cada libro leído. Se tuvo una respuesta favorable, este medio también permitió motivar durante la competencia: videos, ligas para descargar libros, sugerencias de lecturas, información de librerías que continuaban con el servicio en línea; esto con el fin de aprovechar el tiempo durante el aislamiento.

El proyecto de "Lecturatón" fue trabajado bajo la modalidad de investigaciónacción, con el fin de conocer, analizar e ir buscando alternativas de recreación y autoayuda por el aislamiento de la cuarentena vivida en el país y específicamente al interior de la Escuela Normal; pero también se tuvo una meta paralela a esta actividad, que es la del fomento a la lectura en toda la comunidad IByCENECH.

\section{Justificación}

El mes de marzo del año 2020, México como país empezó a vivir una experiencia nueva ante la COVID-19, las generaciones jóvenes solo tenían en sus recuerdos el precedente que se dio años atrás con la pandemia de influenza, nada comparado con lo vivido en la época actual, las formas de trabajo y de vida en general de toda la población se vieron modificadas, los hábitos de las personas cambiaron de una forma abrupta en muchos casos, en otros quizá no lo fueron tanto.

Ante la situación descrita surgió "Lecturatón IByCENECH” con la intención de fomentar hábitos lectores en la comunidad normalista en los días de la cuarentena, considerando que la lectura tiene varias bondades en la vida de un ser humano, debido 
a que además de generar conocimiento, incrementar la cultura, abrir nuevos mundos a través de las experiencias de otros, también puede ser una herramienta que ayude a disminuir el estrés que se genera en un ambiente de aislamiento.

\section{Planteamiento del problema}

Ante la pandemia se presentó un problema mundial derivado de la COVID-19, por el cual se tomaron medidas de contingencia con base en uno de los pilares de la educación. La lectura es una de las competencias para la vida, que se aprende desde nivel básico, se desarrolla y potencializa a lo largo de los años de estudiante, presente en las diferentes situaciones de la vida cotidiana; por lo tanto, es de vital importancia que el docente desarrolle sus habilidades lectoras para fomentarlas dentro del aula.

Durante la formación docente se analizan varios textos, se realizan investigaciones que demandan lecturas complejas, se ven en la necesidad de redactar escritos; tanta es la carga administrativa para los futuros docentes, que se crean una idea errónea de que la lectura es aburrida, este es un problema que se podría generalizar en los estudiantes, incluso durante los primeros años académicos; se interpreta que el leer es por obligación.

Ante esta situación fue que se implementó el proyecto de "Lecturatón", para desarrollar una estrategia, en un primer momento como autoayuda de las personas para enfrentar el aislamiento por el sector salud, pero a la vez fomentar la lectura desde una metodología no impositiva, es decir, desarrollar el gusto por los libros. Es un tanto complejo dar libertad, puesto que hoy en día existen innumerables tipos de textos, porque cada persona es diferente y, al serlo, buscarán diversos temas. Se tomó esto como una ventaja para que, al momento de compartir las reseñas, se pueda enriquecer a todos los participantes en este reto.

Se implementó la lectura no como carga administrativa de trabajo sino como medida terapéutica de autoayuda para atender las necesidades emocionales de la persona, como son: su autoconocimiento a través de los temas de interés; la autorregulación de sus emociones ante el aislamiento; la empatía cuando compartían sus reseñas y la oportunidad de conocer los intereses de otros compañeros; la colaboración al momento de brindar ayuda con textos, páginas de interés, compartir información y, finalmente, potencializar su autonomía al hacer frente a la situación, desarrollándose como lectores activos y creciendo tanto profesional como personalmente. Como medida académica en el desarrollo de la lectura, son los docentes los primeros en brindar el ejemplo de un lector activo, reforzar la comprensión y la mejora del entendimiento de textos.

Los alumnos de la IByCENECH no serían los únicos en estar en aislamiento, por esta razón se contempló también al personal administrativo y docente para participar en este reto, una manera de unificar la comunidad de la institución atendiendo a los cambios que se venían por la contingencia de la COVID-19, creando lazos fuertes para 
enfrentar los desafíos de cambio en la vida cotidiana y fomentar el gusto por la lectura como una estrategia de autoayuda; por tanto, lo que se pretende investigar es: ¿Cómo la lectura se vuelve una herramienta de autoayuda, para enfrentar el aislamiento?

\section{Propósito}

Fomentar la cultura de la lectura en la comunidad normalista, como una respuesta ante las posibles consecuencias psicoemocionales que se puedan presentar durante el aislamiento por la COVID-19, con una duración del 23 de marzo hasta el 17 de junio del 2020.

\section{MarCo teórico}

Leer es un proceso cognitivo complejo que activa estrategias de alto nivel: dotarse de objetivos; establecer y verificar planeaciones; controlar lo que se va leyendo; tomar decisiones en torno a las dificultades o lagunas de comprensión; diferenciar lo que es esencial de la información secundaria. Este proceso requiere necesariamente la participación activa y efectiva del lector. No es un aprendizaje mecánico, ni se realiza todo de una vez: no puede limitarse a un curso de la educación obligatoria (Solé, 2000, p. 88).

Garrido (2006) afirma que solo quien lee mucho llega a ser buen lector. Los conocimientos, las expectativas y las experiencias de cada persona desempeñan un papel decisivo en esta tarea. Para cada lector, la lectura de un mismo texto se vuelve algo personal, al hablar de "personal" es por lo que desarrollamos el autoconocimiento a través de nuestra lectura.

Mejorar la lectura aumenta la capacidad de aprendizaje, favorece el desarrollo del lenguaje, la concentración, el raciocinio, la memoria, la personalidad, la sensibilidad y la intuición, nos muestra la diversidad del mundo y hace más amplios nuestros horizontes. Mejorar la lectura nos ayuda a vivir mejor, y en este caso también nos ayuda a dar respuesta a las demandas emocionales ante el aislamiento por la contingencia.

Los libros son difíciles de leer, entre más significados y descripciones muestren. Harold Bloom (2000) decía: "Leemos para fortalecer nuestra personalidad y averiguar cuáles son sus auténticos intereses", creando la necesidad de adquirir lecturas del mismo género literario, conocer otros autores y ampliar nuestro acervo cultural y lingüístico, lo cual lleva al lector a comprender lecturas más complejas.

Leer correctamente es más que simplemente recorrer con los ojos las palabras de un texto, es establecer un vínculo con el texto que involucra al lector intelectual y emocionalmente. Es desarrollar la facultad de comprender y sentir plenamente un escrito, capacidad que se desarrolla a medida que se ejercita la habilidad intelectual de leer, que es algo mucho más complejo que la sencilla alfabetización, al ser un proceso de adquisición de nueva información, para complementar y formular nuevos conceptos. 
Bloom (2000) hace reflexionar cuando escribe:

No hay una sola manera de leer bien, aunque hay una razón primordial para que leamos. A la información tenemos acceso ilimitado, pero ¿dónde encontramos la sabiduría? Si uno es afortunado, tal vez se tope con un maestro que lo ayude; pero al cabo está solo y debe seguir adelante sin más mediaciones. Leer bien es uno de los mayores placeres que puede proporcionar la soledad, porque, al menos según la experiencia, es el más saludable desde un punto de vista espiritual. Hace que uno se relacione con la alteridad, ya sea la propia, la de los amigos o la de quienes pueden llegar a serlo. La invención literaria es alteridad, y por eso alivia la soledad. Leemos no solo porque nos es imposible conocer a toda la gente que quisiéramos, sino porque la amistad es vulnerable y puede menguar o desaparecer, vencida por el espacio, el tiempo, la falta de comprensión y todas las aflicciones de la vida familiar y pasional.

Existe mala fama de que en México no nos gusta leer, debido a las cifras que confirman que, pese al número de alfabetos, no se cuenta con lectores activos; la mayoría de los habitantes adultos del país saben leer y escribir, eso da una cifra de decenas de millones de personas; tan solo en educación superior existen actualmente casi dos millones de estudiantes. Sin embargo, los tirajes de las principales publicaciones son de unos cuantos miles de ejemplares, ni periódicos, ni revistas, mucho menos libros se editan en un número de ejemplares cercano al de los millones de mexicanos que saben leer y escribir.

De la Torre (1999), en su defensa por el libro, dijo:

El libro, medio y forma más preciso y perfecto por los cuales el pensamiento humano a través de la escritura se conserva y transmite entre los hombres, es a la vez defensa y amenaza. Defensa de la inteligencia, del espíritu, de la capacidad de los seres racionales para expresar su pensamiento, sus ideas preñadas de emociones, de intelecciones explicativas del propio hombre y de su mundo circundante, de juicios en torno de la conducta propia y ajena, y del pensar particular y de los demás, amenaza para quien trata de limitar el pensamiento y su expresión, para quien teme el enjuiciamiento de una conducta reprobable o la condenación de bastardos intereses. Defensa del hombre en su calidad esencial y amenaza contra quien o quienes por cualquier razón se oponen al desarrollo completo e integral de las cualidades humanas.

El fomento a la lectura en la escuela Normal es la llave a la generación de una comunidad pensante, culta, que sea capaz de innovar y proponer alternativas de mejora continua; un grupo de personas que leen de manera habitual tiene una mejor actitud, su cerebro se mantiene activo, sus expectativas ante la vida son altas, el manejo de la información es fluido, la adaptación a nuevos retos es una constante. Por todas estas razones fue implementada e investigada la estrategia "Lecturatón IByCENECH" en los días de aislamiento por la COVID-19.

\section{Metodología}

El estudio es de corte cualitativo bajo el enfoque de investigación-acción, con la finalidad de recuperar y como efecto producir en la comunidad estudiantil desarrollo de habilidades lectoras a través de la implementación de las estrategias, con el 
propósito de dar solución de las necesidades que surgieron ante la pandemia por la COVID-19. El trabajo realizado consistió, como ya se ha mencionado, en involucrar a la comunidad IByCENECH en una estrategia global de lectura en casa, por tanto esta investigación pretende conocer, analizar y buscar alternativas para enfrentar la cuarentena vivida en el país y específicamente al interior de la Escuela Normal-EN-.

\section{Procedimiento}

En esta investigación un grupo de maestros se dio a la tarea de motivar (creando la estrategia) e indagar (documentar, analizar y teorizar) qué nivel de lectura se tiene en la institución, con el fin primeramente de hacer uso de la lectura como técnica de autoayuda en el periodo de cuarentena, pero también se tiene una meta paralela a esta actividad que es la del fomento a la lectura en toda la comunidad IByCENECH.

Se pretendió que la Institución Benemérita y Centenaria Escuela Normal del Estado de Chihuahua creará una vinculación entre la investigación y los hábitos lectores de su población, considerando que hacer investigación contribuye a ampliar el currículo del docente, la cultura del personal administrativo, e incrementar los conocimientos del profesorado en formación. En sí se puede afirmar que la lectura puede mejorar el aprendizaje para todos, siempre y cuando la estrategia se pueda ir adaptando y mejorando en el desarrollo de la actividad (Latorre, 2003).

Las técnicas de recuperación de la información fueron en un primer momento por medio de un formulario digital para conocer los intereses y habilidades lectoras de los participantes, posterior a ello se generó un repositorio con las diferentes reseñas escritas, con una retroalimentación a los participantes; en un tercer momento se hizo el ajuste para trabajar a través de narrativas orales de manera virtual.

El análisis de la información fue una parte relevante para procesar los datos, por lo que se categorizó por medio de las habilidades lingüísticas: escuchar, hablar, leer y escribir, que se lograron identificar con los productos elaborados mediante la reflexión, al hacer la lectura de libros, redacción de narrativas, la expresión oral a través de videos y escuchar al compartir entre los participantes en el grupo.

Diversos autores dan una definición sobre lo que es investigación-acción: Eliot (1993), Kemmis (1984), Lomax (1990), Bartolome (1986) y Lewin (1946), algunos agregan nuevos términos o lo abordan con sinónimos, pero todos llegan al mismo concepto: la investigación-acción consiste en que los profesionales de la educación (docentes) detecten una problemática o necesidad en su contexto la cual sea apta y viable para intervenir buscando una mejora (Latorre, 2003).

De igual manera, los antes mencionados y otros autores han especificado algunas de las características más importantes de la investigación-acción, la cual debe ser participativa, colaborativa, crear comunidades autocríticas; un proceso sistemático de aprendizaje; generar teoría a partir de la práctica, esto implica registrar, recopilar, analizar nuestros propios juicios, reacciones e impresiones en torno a lo que ocurre, 
a través de un diario con reflexiones. La investigación-acción es un proceso político que implica progresivamente cambios que afectan a las personas, realizando análisis críticos de las diversas situaciones, de manera que el número de integrantes de la investigación aumenta gradualmente.

Zuber-Skerritt (1992), además de abordar que sea práctica, participativa, colaborativa e interpretativa, agrega un concepto distinto a los demás: "emancipadora"; se refiere a que en el desarrollo de la intervención todos los actores implicados pueden aportar de la misma forma, sin alguna jerarquización establecida, generando una aportación importante y significativa a la investigación (Latorre, 2003).

Kemmis y McTaggart (1988) puntualizan el propósito de dicha metodología de la siguiente manera: la investigación-acción propone mejorar la educación (no solo impartida en una aula, en esta investigación se fue más allá, se encaminó hacia la comunidad IByCENECH) a través del cambio y aprender a partir de las consecuencias. Este tipo de investigación no se limita a encontrar la explicación de una problemática detectada sino que busca crear estrategias, técnicas, actividades que se puedan aplicar durante la práctica, en este caso el "fomento a la lectura".

Por último, el tipo de investigación-acción que se reconoce en Latorre (2003) y se ejecuta en el presente documento es el que defienden Carr y Kemmis: ser crítica, emancipatoria, con la moderación de los docentes creadores de la estrategia ya descrita.

Latorre (2003) da a conocer cuatro autores con modelos parecidos pero cada uno con diferente organización respecto al proceso para aplicar la investigación acción: Lewin (1946) "se compone de una serie de pasos: planificación, acción y evaluación de la acción. Comienza con una «idea general»” (p. 35). Elliott (1993) toma como punto de partida el modelo de Lewin y lo remodela con tres fases: identificación de una idea general, exploración o planteamiento de las hipótesis de acción y la construcción del plan de acción (Latorre, 2003). Whitehead (1991) establece un espiral de ciclos con los siguientes pasos: sentir o experimentar un problema, imaginar la solución del problema, poner en práctica la solución imaginada, evaluar los resultados de las acciones emprendidas y modificar la práctica a la luz de los resultados (Latorre, 2003).

El cuarto autor es Kemmis (1989), del cual se basa la presente investigación; este se apoya en el modelo de Lewin, "el proceso está integrado por cuatro fases o momentos interrelacionados: planificación, acción, observación y reflexión. Cada uno de los momentos implica una mirada retrospectiva y una intención prospectiva, que forman conjuntamente una espiral autorreflexiva de conocimiento y acción" (Latorre, 2003, p. 35).

Durante esta investigación el proceso, según el ciclo de Kemmis, se trabajó de la siguiente manera: la planificación implicó la creación de un diagnóstico por medio de un formulario digital; basado en los resultados se generó un proyecto con el propósito de dar respuesta a las posibles consecuencias psicoemocionales que se pudieran presentar durante el aislamiento por la COVID-19 a través del fomento a la lectura; 
durante la acción se implementó una serie de estrategias que consisten en lectura de diversos géneros, escritura de reseñas y compartir experiencias con otros. Durante este proceso se realizó la observación con la revisión de los escritos de los participantes, así como las interacciones al compartir sus productos entre los usuarios del grupo cerrado de "Lecturatón IByCENECH"; la reflexión permitió replantear la propuesta para continuar con el ajuste a la dinámica escrita por la lingüística (reseña escrita por el discurso de la reseña), resaltando que todo el proceso se llevó a cabo de manera virtual. Posteriormente se planteará un segundo ciclo para ser trabajado de manera presencial.

\section{Plan de intervención RESUMido}

La presente investigación-acción fue planteada para desarrollarla a través de dos ciclos, el primero de ellos consta de las siguientes actividades: creación del proyecto fue la primera de ellas, en la cual bajo la visión de la maestra Vianey Salinas Porras se conformó el equipo de trabajo para el "Lecturatón IByCENECH”. Se creó el plan de trabajo que dio pie a la segunda actividad, la presentación el proyecto a la junta directiva, esta acción consistió en dar a conocer a las autoridades de la institución el proyecto y las fases de su implementación. Al contar con la aprobación arrancó la tercera actividad, cuyo propósito fue darle difusión por diversos medios digitales al proyecto; aquí cabe mencionar que varias personas de diferentes áreas de trabajo de la EN se involucraron para colaborar, tal es el caso del maestro César Delgado Valles, que apoyó con la fase de inscripción de los participantes. Como etapa final del primer ciclo se creó un grupo de Facebook cerrado, para que los integrantes pudieran compartir las reseñas de los libros que iban leyendo; a su vez la maestra Coynta Becerra Aragonés se encargó de ir motivando a los participantes con comentarios alentadores a sus envíos.

El segundo ciclo aún no se efectúa, debido a que fue planeado con actividades de tipo presencial al terminar el periodo de aislamiento en la ciudad; este segundo ciclo consta también de varias actividades tales como: premiación a las personas que mayor número de libros hayan leído, grupos focales para medir la efectividad del proyecto, creación de un compendio de reseñas que será publicado en la comunidad IByCENECH a través de sus plataformas y, por último, presentación de resultados al consejo directivo.

\section{CONCLUSIÓN}

Con este proyecto se tuvo la posibilidad de hacer del aislamiento en casa en tiempos de la COVID-19 una dinámica de autoayuda y fomento a la lectura para los involucrados, a través de la creación e implementación de una serie de estrategias que permitieron a los participantes no solo leer sino hacer un análisis de lo leído, plasmado de manera escrita en un primer momento, para posteriormente hacer el ajuste de presentar las reseñas en video en el grupo cerrado de Facebook; aquí se dio una interacción entre 
los lectores, generando un ambiente armonioso y nutrido de experiencias únicas, por lo que se puede afirmar que los resultados fueron alentadores, se erradicó el mito de que la lectura es aburrida por el reflejo de las participaciones y comentarios que se hacían constantemente en la red social. Es así que se fomenta la lectura, la escritura y el análisis pero, sobre todo, este proyecto se convirtió en una oportunidad para disminuir el estrés generado por el aislamiento; brindar acompañamiento y retroalimentación constante mantuvo la motivación, tal es el caso que compartían libros entre sí, promovieron acervos de distintas fuentes o eventos relacionados con los mismos, sin ser parte de la dinámica inicial de "Lecturatón", dando como resultado una comunidad lectora a distancia, lo cual hace ver que el proyecto superó las expectativas planteadas en el propósito, el cual consistió principalmente en fomentar la cultura de la lectura en la comunidad normalista, dando seguimiento hasta el día de hoy a nuevas actividades de interacción lectora; este proceso se analizó por medio del método de investigación-acción a través de los ciclos de Kemmis, permitiendo recomponer las acciones para seguir en esta constante generación de espacios lectores.

\section{REFERENCIAS}

Baker, L., y Brown, A. (1984). Cognitive monitoring in reading. En J. Flood (ed.) Understandindg reading comprehension. Newark: IRA.

Bloom, H. (2000). Cómo y por qué leer. Barcelona: Anagrama.

Cooper, J. D. (1999). Cómo mejorar la comprensión lectora (3a. ed.). España: Visor.

De la Torre Villar, E. (1991). Elogio y defensa del libro (4a. ed.. México: UNAM.

Freire, P. (1991). La importancia de leer y el proceso de liberación (8a. ed.. México: Siglo XXI.

Garrido, F. (2006). Cómo leer (mejor) en voz alta. Una guía para contagiar la afición a leer. México: Fundación Mexicana para el Fomento de la Lectura.

Goldin, D. (ed) (2006). Encuesta Nacional de Lectura. Informes y evaluaciones. México.

Jitrik, N. (1998). Lectura y cultura (3a. ed.). México: UNAM.

Latorre, A. (2003). La investigación-acción. Conocer y caminar la práctica educativa. Barcelona: Grao.

Solé, I. (2000). La adquisición de la lectura en la escuela primaria. México: SEP.

Cómo citar este artículo:

Salinas Porras, V., Oros Macías, R., y Becerra Aragonés, C. (2020). Lecturatón en la IByCENECH. RECIE. Revista Electrónica Científica de Investigación Educativa, 5(1), pp. 419-427. doi: doi.org/10.33010/recie.v5i1.1071. 